\title{
CÁC YẾU TỐ ẢNH HƯỞNG ĐẾN Ý ĐỊNH KHỞI NGHIẸP KINH DOANH CỦA SINH VIÊN KHỐI NGÀNH KINH TẾ CÁC TRƯờnG ĐẠI HỌC TẠI THÀNH PHỐ HỒ CHÍ MINH
}

\section{FACTORS AFFECT THE START-UP INTENTION OF UNIVERSITY STUDENTS IN ECONOMICS AREA AT HO CHI MINH CITY}

\section{Nguyễn Xuân Hiệp, Trần Hà Thanh, Nguyễn Thị Yến Nhi ${ }^{1}$}

Ngày nhận bài: 11/11/2018 Ngày chấp nhận đăng: 22/11/2018 Ngày đăng: 05/6/2019

\section{Tóm tắt}

Nghiên cứu này nhằm xác định các yếu tố ảnh hưởng đến ý định khởi nghiệp kinh doanh của sinh viên khối ngành kinh tế các trường đại học tại Thành phố Hồ Chí Minh (TP.HCM), từ đó đề xuất các hàm ý chính sách nhằm thúc đẩy tinh thần khởi nghiệp kinh doanh của sinh viên. Mô hình nghiên cứu đề xuất kế thừa mô hình nghiên cứu của Ambad và Damit (2016). Dữ liệu nghiên cứu được thu thập từ 430 sinh viên năm cuối, khối ngành kinh tế của 10 trường Đại học tại TP.HCM có tỷ lệ sinh viên khởi nghiệp cao sau khi tốt nghiệp và được kiểm định bằng phân tích hồi quy tuyến tính bội. Kết quả nghiên cứu cho thấy, các yếu tố ảnh hưởng đến ý định khởi nghiệp kinh doanh của sinh viên khối ngành kinh tế các trường Đại học tại TP.HCM (được sắp xếp theo trình tự mức độ quan trọng từ cao xuống thấp) bao gồm: Giáo dục kinh doanh (GDKD); Chuẩn chủ quan (CCQA); Môi trường khởi nghiệp (MTKN); Đặc điểm tính cách (DDTC) và Nhận thức tính khả thi (NTKT). Tì khóa: Ý định khởi nghiệp kinh doanh, sinh viên khối ngành kinh tế, môi trường khởi nghiệp.

\begin{abstract}
This study aims to identify the factors these affect the start-up intention of university student in economics area at Ho Chi Minh City. From there, policy implications are introduced in order to promote the entrepreneurial spirit of the students. The proposed model inherits the research model of Ambad and Damit (2016). The research data was collected from 430 economic final-year students from 10 universities where have the highest rate of start-up students in Ho Chi Minh area and and it is tested by Multiple Linear Regression Analysis Model. The results show that the factors affecting the intention of startups of university student in economics area at Ho Chi Minh City (arranged in order of importance from highest to lowest) include: Business education (GDKD); Subjective standards (CCQA); Startup Environment (MTKN); Personality Characteristics (DDTC) and Perception of Feasibility (NTKT),...
\end{abstract}

Keywords: Start-up intention, students in economics, startup environment.

\footnotetext{
${ }^{1}$ Trường Đại học Tài chính - Marketing
} 


\section{1. Đặt vấn đề}

Ngày nay, kinh doanh trở thành nhân tố quyết định sự phát triển đối với tất cả các quốc gia. Vì thế, một trong những chiến lược tốt nhất để phát triển kinh tế của đất nước và duy trì khả năng cạnh tranh trước xu hướng toàn cầu hóa ngày càng gia tăng là phát triển tinh thần kinh doanh (Schaper và Volery, 2004; Venkatachalam và Waqif, 2005). Trong đó, nhiều kết quả nghiên cứu (ví dụ: Tam, 2009; Ooi và cộng sự, 2011) đã chứng minh giáo dục kinh doanh có tầm quan trọng trong việc khơi gợi cảm hứng cho sinh viên hướng đến kinh doanh và các tổ chức giáo dục bậc cao là các cơ sở phát triển và khai thác đội ngũ doanh nhân tiềm năng.

Tại Việt Nam, tinh thần doanh nhân và khởi nghiệp được nhân lên trong những năm gần đây, sau khi Chính phủ phát động phong trào khởi nghiệp và lấy năm 2016 là năm quốc gia khởi nghiệp. Nhiều trường đại học đã đưa giáo dục khởi nghiệp vào chương trình đào tạo, thậm chí xây dựng thành một ngành, chuyên ngành đào tạo. Tuy nhiên, tại TP.HCM - Trung tâm kinh tế - thương mại và khoa học - công nghệ lớn nhất của cả nước, với hơn 80 trường đại học, cao đẳng và khoảng gần một triệu sinh viên, nhưng số lượng và tỷ lệ sinh viên sau tốt nghiệp khởi nghiệp còn rất thấp, ngay cả đối tượng sinh viên khối ngành kinh tế. Đây chính là lý do để tác giả thực hiện nghiên cứu này, nhằm xác định các yếu tố ảnh hưởng đến ý định khởi nghiệp kinh doanh của sinh viên khối ngành kinh tế các trường Đại học tại TP.HCM, từ đó đề xuất các hàm ý chính sách nhằm thúc đẩy tinh thần khởi nghiệp kinh doanh của sinh viên trong giai đoạn hiện nay.

\section{Cơ sở lý thuyết và mô hình nghiên cứu}

Nghiên cứu này được thực hiện dựa vào:

- Thuyết hành động hợp lý (TRA) của Ajzen và Fishbein (1975); Lý thuyết về hành vi dự định (TPB) - Ajzen (1991); Mô hình sự kiện khởi nghiệp của Shapero và Sokol (1982); Mô hình cấu trúc ý định kinh doanh của Luthje và Franke (2003).

- Các nghiên cứu về các yếu tố ảnh hưởng đến ý định khởi nghiệp, bao gồm: nghiên cứu của Autio và cộng sự (2001) về mô hình ý định kinh doanh giữa sinh viên châu Âu và Bắc Mỹ; nghiên cứu của Lĩnán và cộng sự (2011) về các yếu tố ảnh hưởng đến ý định khởi nghiệp của sinh viên Tây Ban Nha; nghiên cứu của Karali (2013) về mô hình tác động của giáo dục kinh doanh đến ý định kinh doanh; nghiên cứu của Ambad và Dami (2016) về các yếu tố ảnh hưởng đến ý định khởi nghiệp của sinh viên tại Malaysia; nghiên cứu của Phan Anh Tú và Giang Thị Cẩm Tiên (2015) về ý định khởi nghiệp của sinh viên Đại học Cần Thơ; nghiên cứu của Đỗ Thị Hoa Liên (2016) về các nhân tố ảnh hưởng đến ý định khởi nghiệp kinh doanh của sinh viên ngành quản trị kinh doanh trường Đại học Lao động - Xã hội.

Tổng kết các lý thuyết về ý định hành vi và các nghiên cứu trên đây cho thấy, mô hình nghiên cứu các yếu tố ảnh hưởng đến ý định khởi nghiệp kinh doanh đều dựa vào thuyết hành vi hoạch định (TPB) của Ajzen (1991). Vì thế, phần lớn các nghiên cứu đều xác định các yếu tố chính ảnh hưởng đến ý định khởi nghiệp kinh doanh của sinh viên bao gồm: (1) Thái độ đối với hành vi khởi nghiệp; (2) Chuẩn chủ quan; (3) Nhận thức tính khả thi. Trong đó, 
thái độ đối với hành vi khởi nghiệp, theo Luthje và Franke (2003) được giải thích bởi: nhu cầu thành đạt; xu hướng chấp nhận rủi ro và quỹ tích kiểm soát nội bộ (gọi chung là đặc điểm tính cách); hoặc theo Karali (2013) là chương trình giáo dục kinh doanh.

Ngoài các yếu tố trên, theo Luthje và Franke (2003), ý định khởi nghiệp kinh doanh còn chịu ảnh hưởng của các tác nhân từ bên ngoài (gọi là hỗ trợ nhận thức) nhằm củng cố và gia tăng ý định khởi nghiệp kinh doanh. Nghiên cứu của Grimaldi và Gradi (2005); Radas và Bozic (2009) cho rằng, môi trường khởi nghiệp có ảnh hưởng tích cực đến kết quả kinh doanh của doanh nghiệp vừa khởi nghiệp. Radas và Bozic (2009); Ambad và Damit (2016) cho thấy, các chính sách của Chính phủ hỗ trợ các nhà khởi nghiệp kinh doanh năng động, cũng như khuyến khích các doanh nghiệp trong giai đoạn đầu sau khi khởi nghiệp.

Kết quả nghiên cứu định tính khẳng định, sự hỗ trợ từ phía môi trường khởi nghiệp là tác nhân quan trọng góp phần hình thành và thúc đẩy ý định khởi nghiệp kinh doanh của sinh viên. Vì, với đối tượng sinh viên, ngoài những kiến thức lý thuyết được trang bị trong nhà trường, cùng ý chí và khát vọng thành đạt của tuổi trẻ, thì những điều kiện khác để khởi nghiệp kinh doanh như: sự hỗ trợ về nguồn lực tài chính và những trải nghiệm từ thực tiễn hoạt động quản lý, điều hành doanh nghiệp, cũng như khả năng thích ứng với những biến động của thị trường, dường như còn là khoảng trắng. Nghĩa là, họ rất cần sự hỗ trợ đắc lực từ môi trường khởi nghiệp, mà theo Grimaldi và Gradi (2005); Radas và Bozic (2009) đó là việc tiếp cận các nguồn lực tài chính; các chính sách hỗ trợ của Chính phủ; sự hỗ trợ tư vấn, đào tạo về khởi nghiệp từ các tổ chức phi Chính phủ. Trên cơ sở đó, nhóm tác giả đề xuất mô hình nghiên cứu gồm 05 yếu tố, kế thừa từ mô hình nghiên cứu của Lĩnán và cộng sự (2011), Ambad và Damit (2016), trong đó yếu tố cơ chế, chính sách của Chính phủ được thay thế bằng yếu tố môi trường khởi nghiệp.

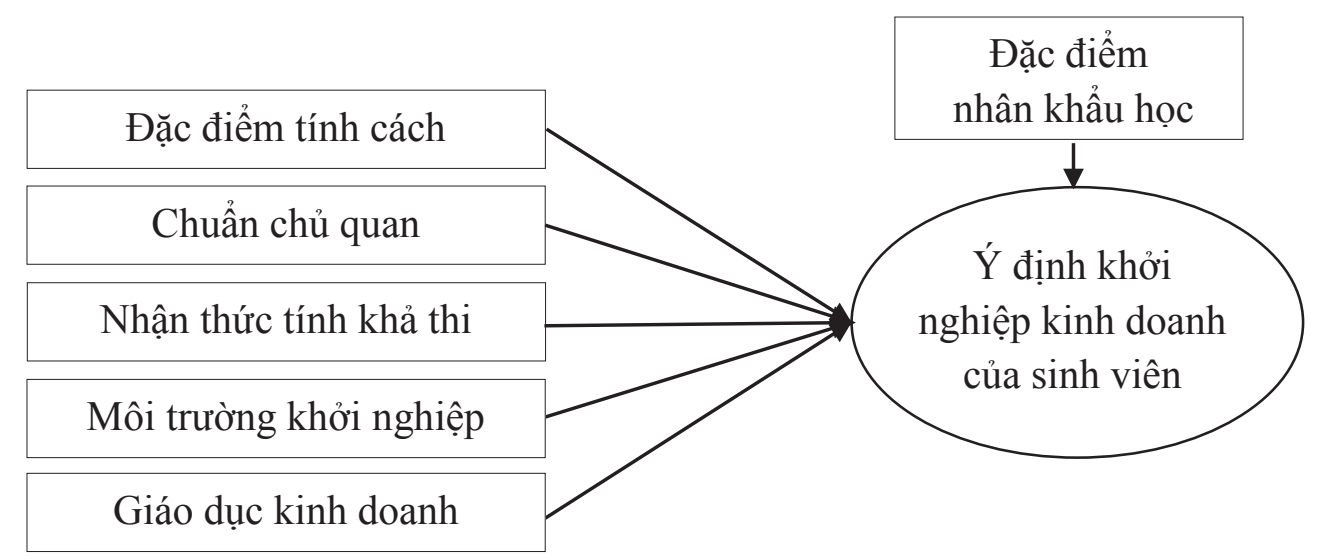

\section{Hình 1. Mô hình nghiên cứu của nhóm tác giả}

Trong đó:

- Đặc điểm tính cách: nói lên tính cách của doanh nhân. Theo Luthje và Franke (2003), đặc điểm tính cách ảnh hưởng đến ý định khởi nghiệp trên 3 khía cạnh: (1) Nhu cầu thành đạt phản ánh mong muốn thành đạt của cá nhân; (2)
Quỹ tích kiểm soát nội bộ thể hiện mức độ tự tin và quyền lực của cá nhân trong việc kiểm soát hành vi kinh doanh và kết quả của hành vi đó; (3) Chấp nhận rủi ro thể hiện sự sẵn sàng chấp nhận những tổn thất do rủi ro gây ra trong quá trình khởi nghiệp. 
Nghiên cứu của Luthje và Franke (2003); Lĩnán và cộng sự (2011); Ambad và Damit (2016) đều tìm thấy, đặc điểm tính cách có ảnh hưởng trực tiếp và tích cực đến ý định tham gia khởi nghiệp kinh doanh.

H1: Đặc điểm tính cách ảnh hương cùng chiều đến ý định khởi nghiệp kinh doanh của sinh viên.

- Chuẩn chủ quan: là nhận thức về những áp lực từ phía xã hội thể hiện sự ủng hộ, hay phản đối người có ý định thực hiện hành vi. Nó bao gồm các ảnh hưởng bên trong là ý kiến từ gia đình, bạn bè, đồng nghiệp và các ảnh hưởng bên ngoài là các trào lưu xã hội.

Các nghiên cứu của Karali (2013; Lĩnán và cộng sự (2011); Ambad và Damit (2016) tìm thấy, chuẩn chủ quan có ảnh hưởng trực tiếp và tích cực đến ý định tham gia khởi nghiệp kinh doanh.

H2: Chuẩn chủ quan ảnh hưởng cùng chiều đến ý định khởi nghiệp kinh doanh của sinh viên.

- Nhận thức tính khả thi: phản ánh nhận thức của cá nhân về mức độ dễ dàng hay khó khăn; có bị kiểm soát, hạn chế hay không khi thực hiện hành vi (Ajzen 1991, tr. 183); là mức độ tự tin của một cá nhân về khả năng thực hiện các hành vi (Ajzen, 2006). Trong nghiên cứu này là cảm nhận của cá nhân về khả năng khởi nghiệp.

Karali (2013); Ambad và Damit (2016) cho thấy nhận thức tính khả thi có ảnh hưởng trực tiếp đến ý định hành vi. Autio và cộng sự (2001) chứng minh nhận thức tính khả thi nổi lên như là yếu tố quyết định quan trọng nhất ảnh hưởng tích cực đến ý định kinh doanh.

H3: Nhận thức tính khả thi ảnh hương cùng chiều đến ý định khởi nghiệp kinh doanh của sinh viên.
- Giáo duc kinh doanh (giáo dục tinh thần kinh doanh): là những nội dung giáo dục liên quan đến chương trình, các bài giảng ngoại khóa, hoặc các khóa học cung cấp cho sinh viên những kiến thức, kỹ năng và thái độ để theo đuổi sự nghiệp kinh doanh (Ambad và Damit, 2016).

Lĩnán và cộng sự (2011); Ambad và Damit (2016) đã kiểm chứng giáo dục kinh doanh có mối liện hệ tích cực đến ý định kinh doanh; giáo dục tinh thần kinh doanh là một phương tiện hiệu quả trong việc gây cảm hứng sinh viên có ý định khởi nghiệp kinh doanh.

H4: Giáo dục kinh doanh ảnh hương cùng chiều với ý định khởi nghiệp kinh doanh của sinh viên.

- Môi truờng khởi nghiệp: là tập hợp các yếu tố quy định khả năng tiếp cận các nguồn lực tài chính; các chính sách hỗ trợ của Chính phủ; sự hỗ trợ tư vấn, đào tạo về khởi nghiệp từ các tổ chức phi Chính phủ; sự hỗ trợ tiếp cận thị trường, cùng các chuẩn mực văn hóa thúc đẩy ý định khởi nghiệp (Grimaldi và Gradi, 2005; Radas và Bozic, 2009).

Radas và Bozic (2009); Ambad và Damit (2016) cho thấy các chính sách của Chính phủ hỗ trợ cho việc xuất hiện các nhà khởi nghiệp kinh doanh năng động, cũng như khuyến khích các doanh nghiệp trong giai đoạn đầu sau khi khởi nghiệp

H5: Môi truờng khởi nghiệp ảnh hwởng cùng chiều với ý định khởi nghiệp kinh doanh của sinh viên.

Ngoài 05 các yếu tố trên đây, Autio và cộng sự (2001); Yordanova và Tarrazon (2010) đã kiểm chứng có sự khác biệt về ý định khởi nghiệp kinh doanh theo các đặc điểm nhân khẩu học (giới tính, tuổi, trình độ học vấn, gia đình, khóa học kinh doanh, v.v.). 
H6: Có sự khác nhau về ý định khởi nghiệp của sinh viên theo các đặc điểm nhân khẩu học của sinh viên.

\section{Phương pháp nghiên cứu}

Quá trình nghiên cứu gồm hai giai đoạn là nghiên cứu sơ bộ và nghiên cứu chính thức:

- Nghiên cứu sơ bộ là một nghiên cứu định tính được thực hiện bằng kỹ thuật thảo luận nhóm tập trung (gồm 2 nhóm; 01 nhóm cựu sinh viên đã khởi nghiệp năm đầu sau khi tốt nghiệp; 01 nhóm sinh viên năm cuối có ý định khởi nghiệp kinh doanh, mỗi nhóm 10 người), để thẩm định mô hình các yếu tố ảnh hưởng đến ý định khởi nghiệp kinh doanh của sinh viên được đề xuất trên đây và thang đo các yếu tố này.

- Nghiên cứu chính thức là một nghiên cứu định lượng nhằm đánh giá độ tin cậy và giá trị của thang đo các khái niệm nghiên cứu; kiểm định mô hình lý thuyết và các giả thuyết nghiên cứu; kiểm định sự khác biệt về các yếu tố ảnh hưởng đến ý định khởi nghiệp kinh doanh của sinh viên theo các đặc điểm nhân khẩu xã hội học của sinh viên. Kích thước mẫu là 430, được chọn bằng phương pháp lấy mẫu thuận tiện đối với sinh viên đang học năm cuối, khối ngành kinh tế của 10 trường đại học tại TP.HCM có số lượng và tỷ lệ sinh viên khởi nghiệp cao trong những năm đầu sau khi tốt nghiệp (bảng 1).

Bảng 1. Thông tin mẫu nghiên cứu

\begin{tabular}{|c|c|c|c|}
\hline \multicolumn{2}{|r|}{ Cơ cấu mẫu nghiên cứu } & $\begin{array}{c}\text { Số lượng } \\
\text { (người) }\end{array}$ & $\begin{array}{l}\text { Tỷ lệ } \\
(\%)\end{array}$ \\
\hline \multirow{5}{*}{$\begin{array}{l}\text { Các Trường } \\
\text { ĐH công lập }\end{array}$} & Trường ĐH Kinh tế TP.HCM & 66 & 15,3 \\
\hline & Trường ĐH Kinh tế - Luật & 65 & 15,1 \\
\hline & Trường ĐH Tài chính - Marketing & 52 & 12,1 \\
\hline & Trường ĐH Ngoại Thương TP.HCM & 51 & 11,9 \\
\hline & Trường ĐH Ngân hàng. TP.HCM & 41 & 9,5 \\
\hline \multirow{6}{*}{$\begin{array}{l}\text { Các Trường } \\
\text { ĐH dân lập }\end{array}$} & Trường ĐH Công nghệ TP.HCM & 32 & 7,4 \\
\hline & Trường ĐH Hoa Sen & 31 & 7,2 \\
\hline & Trường ĐH Văn Lang & 30 & 7,0 \\
\hline & Trường ĐH FPT & 31 & 7,2 \\
\hline & Trường ĐH Văn Hiến & 31 & 7,2 \\
\hline & & 430 & 100,0 \\
\hline \multirow{2}{*}{ Giới tính } & Nữ & 274 & 63,7 \\
\hline & Nam & 156 & 36,3 \\
\hline \multirow{2}{*}{$\begin{array}{l}\text { Hộ khẩu } \\
\text { thường trú }\end{array}$} & Tại các thành phố & 180 & 41,9 \\
\hline & Các địa phương khác & 250 & 58,1 \\
\hline \multirow{2}{*}{$\begin{array}{l}\text { Nghề nghiệp } \\
\text { của bố mẹ }\end{array}$} & Chủ cơ sở kinh doanh & 142 & 33,0 \\
\hline & Nghề nghiệp khác & 288 & 67,0 \\
\hline \multicolumn{2}{|c|}{ Tổng cộng } & 430 & 100,0 \\
\hline
\end{tabular}




\section{Kết quả nghiên cứu và thảo luận}

Kết quả nghiên cứu định tính khẳng định, các yếu tố ảnh hưởng đến ý định khởi nghiệp kinh doanh của sinh viên khối ngành kinh tế được nhóm tác giả đề xuất trên đây (Hình 1) là những yếu tố chính ảnh hưởng đến ý định khởi nghiệp kinh doanh của sinh viên, đồng thời phát triển thang đo các khái niệm nghiên cứu (thang đo Likert 7 bậc từ $1 \div 7 ; 1$ là hoàn toàn không đồng ý; 7 là hoàn toàn đồng ý) gồm 28 biến quan sát (Bảng 2).

\section{Bảng 2: Thang đo các khái niệm nghiên cứu}

\begin{tabular}{|l|c|c|l|}
\hline Khái niệm nghiên cứu & Ký hiệu & Số biến quan sát & \multicolumn{1}{|c|}{ Nguồn } \\
\hline Đặc điểm tính cách & DDTC & 5 & Lĩnán và cộng sự (2011) \\
\hline Chuẩn chủ quan & CCQA & 4 & Lĩnán và cộng sự (2011) \\
\hline Nhận thức tính khả thi & NTKT & 5 & $\begin{array}{l}\text { Autio và cộng sự (2001); Ambad và } \\
\text { Damit (2016) }\end{array}$ \\
\hline Giáo dục kinh doanh & GDKD & 5 & $\begin{array}{l}\text { của Lĩnán và cộng sự (2011) và Ambad } \\
\text { và Damit (2016) }\end{array}$ \\
\hline Môi trường khởi nghiệp & MTKN & 5 & $\begin{array}{l}\text { Grimaldi và Gradi (2005); Radas và } \\
\text { Bozic (2009); Ambad và Damit (2016) }\end{array}$ \\
\hline Ý định khởi nghiệp & YDKN & 4 & $\begin{array}{l}\text { Lĩnán và cộng sự (2011); Ambad và } \\
\text { Damit (2016) }\end{array}$ \\
\hline
\end{tabular}

(Nguồn: Kết quả nghiên cúu của tác giả)

Kết quả Cronbach's alpha, sau khi loại biến NTKT5 (Bạn có đủ khả năng trở thành một doanh nhân thành đạt) của thang đo Nhận thức tính khả thi có tương quan biến tổng $(=0,282)$ không đạt yêu cầu $(>0,3)$, thang đo các khái niệm nghiên cứu đều đạt độ tin cậy $(>0,6)$.

Kết quả phân tích nhân tố khám phá (EFA) các biến độc lập và biến phụ thuộc bằng phương pháp trích Principal components và phép quay Varimax cho thấy:

- Sau khi loại biến CCQA4 (Nhà nước có các chính sách khuyến khích sinh viên khởi nghiệp) của thang đo yếu tố Chuẩn chủ quan (có hệ số tải nhân tố $=0,440<0,5$ và chênh lệch hệ số nhân tố < 0,3 (0,440 - 0,362), 22 biến quan sát (còn lại) đo lường 5 yếu tố ảnh hưởng đến ý định khởi nghiệp được rút trích vào 05 nhân tố nguyên gốc với hệ số $\mathrm{KMO}=0,919$ và Sig. $=0,000$; phương sai trích $=63,146 \%$, tại Eigenvalue $=1,112$, đồng thời tất cả các biến đều có hệ số tải nhân tố đạt yêu cầu $(>0,5)$.

- 4 biến quan sát của thang đo biến phụ thuộc (ý định khởi nghiệp kinh doanh của sinh viên) được rút trích vào cùng một nhân tố với hệ số $\mathrm{KMO}=0,822$ và $\mathrm{Sig}=0,000$; phương sai trích $=74,896 \%$, tại Eigenvalue $=2,996$, đồng thời tất cả biến đều có hệ số tải nhân tố đạt yêu cầu $(>0,5)$. Chứng tỏ, EFA các biến độc lập và biến phụ thuộc là phù hợp và có thể sử dụng kết quả này cho phân tích hồi qui ở bước tiếp theo.

Kết quả phân tích hồi qui thu được $\mathrm{R}^{2}$ điều chỉnh $=0,606$; giá trị kiểm định $\mathrm{F}=90,733$ với $\operatorname{Sig}=0,000$; các hệ số hồi quy $\mathrm{B}$ và Beta đều $>0$, các giá trị kiểm định $\mathrm{t}$ đều có ý nghĩa 
thống kê (bảng 3); kết quả kiểm tra các vi phạm giả định của mô hình hồi qui đều không bị vi phạm. Chứng tỏ:

- Mô hình hồi quy được dự đoán phù hợp với dữ liệu thị trường và giải thích được $60,6 \%$ sự biến thiên của ý định khởi nghiệp kinh doanh của sinh viên.

- Các giả thuyết H1, H2, H3, H4, H5 được chấp nhận và phương trình hồi quy có dạng:
YDKN $=-1,173+0,205 *$ DDTC

$$
\begin{aligned}
& +0,254 * \mathrm{CCQA}+0,170 * \mathrm{NTKT} \\
& +0,336 * \mathrm{GDKD}+0,276 * \mathrm{MTKN}
\end{aligned}
$$

- Mức độ quan trọng của các yếu tố ảnh hưởng được sắp xếp theo thứ tự từ cao xuống thấp bao gồm: Giáo dục kinh doanh (GDKD); Chuẩn chủ quan (CCCQ); Môi trường khởi nghiệp (MTKN); Đặc điểm tính cách (DDTC) và Nhận thức tính khả thi (NTKT).

\begin{tabular}{|c|c|c|c|c|c|c|c|}
\hline \multirow{2}{*}{$\begin{array}{l}\text { Mô } \\
\text { hình }\end{array}$} & \multicolumn{2}{|c|}{$\begin{array}{l}\text { Hệ số hồi qui } \\
\text { chưa chuẩn hóa }\end{array}$} & \multirow{2}{*}{$\begin{array}{c}\text { Hệ số hồi } \\
\text { qui chuẩn } \\
\text { hóa }\end{array}$} & \multirow{2}{*}{$\begin{array}{l}\text { Giá trị } \\
\text { kiểm } \\
\text { định } \mathrm{t}\end{array}$} & \multirow{2}{*}{$\begin{array}{c}\text { Mức } \\
\text { ý } \\
\text { nghĩa }\end{array}$} & \multicolumn{2}{|c|}{ Thống kê đa cộng tuyến } \\
\hline & B & $\begin{array}{c}\text { Độ lệch chuẩn } \\
\text { của sai số }\end{array}$ & & & & $\begin{array}{c}\text { Độ chấp } \\
\text { nhận }\end{array}$ & $\begin{array}{l}\text { Hệ số phóng đại } \\
\text { phương sai (VIF) }\end{array}$ \\
\hline (Constant) & -1.173 & 0,257 & & $-4,566$ & 0,000 & & \\
\hline DDTC & 0,205 & 0,050 & 0,163 & 4,058 & 0,000 & 0,568 & $1 ., 761$ \\
\hline CCQA & 0,254 & 0,043 & 0,225 & 5,940 & 0,000 & 0,638 & 1,566 \\
\hline NTKT & 0,170 & 0,042 & 0,158 & 4,058 & 0,000 & 0,602 & 1,661 \\
\hline GDKD & 0,336 & 0,046 & 0,284 & 7,289 & 0,000 & 0,603 & 1,659 \\
\hline MTKN & 0,276 & 0,056 & 0,202 & 4,967 & 0,000 & 0,555 & 1,803 \\
\hline
\end{tabular}

Bảng 2. Các thông số của mô hình hồi qui

(Nguồn: Kết quả phân tích dũ liệu của tác giả)

Kết quả kiểm định chưa tìm thấy sự khác biệt về ý định khởi nghiệp kinh doanh của sinh viên khối ngành kinh tế các trường đại học tại TP.HCM theo các đặc điểm: loại hình trường đại học; giới tính; hộ khẩu thường trú; nghề nghiệp của bố mẹ sinh viên. Nghĩa là, giả thuyết H6 (có sự khác biệt về ý định khởi nghiệp của sinh viên khối ngành kinh tế các trường đại học tại TP.HCM theo các đặc điểm nhân khẩu học của sinh viên bị bác bỏ.

Như vậy, kết quả nghiên cứu này tương đồng với nghiên cứu của Lĩnán và cộng sự (2011), nhưng bổ sung yếu tố môi trường khởi nghiệp; tương đồng với nghiên cứu Ambad và Damit (2016), trong đó yếu tố cơ chế, chính sách của
Chính phủ được thay thế bằng môi trường khởi nghiệp. Yếu tố Giáo dục kinh doanh (GDKD); Chuẩn chủ quan (CCQA) có ảnh hưởng mạnh đến ý định khởi nghiệp của sinh viên là tương đồng với nghiên cứu của Lĩnán và cộng sự (2011), Ambad và Damit (2016); Phan Anh Tú và Giang Thị Cẩm Tiên (2015); Đỗ Thị Hoa Liên (2016). Yếu tố môi trường khởi nghiệp có ảnh hưởng mạnh đến ý định khởi nghiệp kinh doanh của sinh viên khối ngành kinh tế là tương đồng với nghiên cứu của Grimaldi và Gradi (2005); Radas và Bozic (2009). Nghiên cứu này chưa tìm thấy sự khác biệt về ý định khởi nghiệp của sinh viên khối ngành kinh tế các trường đại học tại TP.HCM theo các đặc điểm nhân khẩu 
xã hội học là tương đồng với Ambad và Damit (2016); Đỗ Thị Hoa Liên (2016). Tất cả những sự so sánh này, chứng tỏ kết quả nghiên cứu này đáng tin cậy.

\section{Bảng 4. So sánh mức độ ảnh hưởng và giá trị trung bình của các yếu tố ảnh hưởng đến ý định khởi nghiệp kinh doanh của sinh viên}

\begin{tabular}{|l|c|c|c|c|}
\hline \multicolumn{1}{|c|}{ Nhân tố } & Mức độ quan & Giá trị & Giá trị có nhiều lựa & Độ lệch \\
chọn nhất (Mod) & chuẩn \\
\cline { 4 - 5 } Giáo dục kinh doanh & 0,284 & 4,632 & 5 & 0,83199 \\
Chuẩn chủ quan & 0,225 & 4,748 & 5 & 0,87074 \\
\hline Môi trường khởi nghiệp & 0,202 & 5,423 & 6 & 0,71984 \\
\hline Đặc điểm tính cách & 0,163 & 5,535 & 6 & 0,78399 \\
\hline Nhận thức tính khả thi & 0,158 & 5,249 & 5 & 0,91558 \\
\hline
\end{tabular}

(Nguồn: Kết quả phân tích dũ liệu của tác giả)

Tuy nhiên, ở thời điểm hiện tại chưa có sự tương thích giữa mức độ quan trọng của các yếu tố ảnh hưởng đến ý định khởi nghiệp kinh doanh của sinh viên khối ngành kinh tế các trường đại học tại TP.HCM và giá trị trung bình của chúng. Đó là các yếu tố có ảnh hưởng mạnh đến ý định khởi nghiệp kinh doanh lại chưa được sinh viên đánh giá cao và ngược lại (bảng 4). Chứng tỏ các yếu tố ảnh hưởng đến ý định khởi nghiệp kinh doanh của sinh viên khối ngành kinh tế các trường đại học tại TP.HCM chưa được phát triển dựa trên cơ sở khoa học của chúng.

\section{Kết luận và hàm ý chính sách, quản trị}

Mô hình nghiên cứu các yếu tố ảnh hưởng đến ý định khởi nghiệp kinh doanh của sinh viên khối ngành kinh tế các trường đại học tại TP.HCM được đề xuất kế thừa từ mô hình nghiên cứu của Lĩnán và cộng sự (2011), Ambad và Damit (2016). Kết quả nghiên cứu cho thấy, mô hình kiểm định phù hợp với mô hình nghiên cứu đề xuất, trong đó mức độ quan trọng của các yếu tố ảnh hưởng được sắp xếp theo thứ tự từ cao xuống thấp bao gồm: Giáo dục kinh doanh (GDKD); Chuẩn chủ quan (CCCQ); Môi trường khởi nghiệp (MTKN);
Đặc điểm tính cách (DDTC) và Nhận thức tính khả thi (NTKT). Tuy nhiên, mô hình nghiên cứu chỉ giải thích được $60,6 \%$ biến thiên của ý định khởi nghiệp kinh doanh của sinh viên. Chứng tỏ, khả năng còn có những yếu tố khác cũng tham gia giải thích ý định khởi nghiệp kinh doanh của sinh viên nhưng chưa được cô đọng trong mô hình nghiên cứu.

Từ kết quả này, để thúc đẩy tinh thần khởi nghiệp kinh doanh của sinh viên khối ngành kinh tế các trường đại học tại TP.HCM trong giai đoạn hiện nay, các nhà hoạch định chính sách và các trường đại học cần tập trung vào các hàm ý chính sách và quản trị sau đây.

Một là, nâng cao vai trò và chất lượng giáo dục kinh doanh trong các trường đại học bằng cách:

- Đưa các khóa học doanh nhân vào chương trình bắt buộc của trường đại học đào tạo khối ngành kinh tế, đồng thời gia tăng thời lượng và nâng cao chất lượng chương trình giáo dục kinh doanh trong các trường đại học.

- Xác lập danh mục, mã ngành, chuyên ngành quản trị khởi nghiệp trong các trường đại học khối ngành kinh tế. Trong đó, chương trình 
đào tạo quản trị khởi nghiệp cần phải trực tiếp trang bị các tố chất kinh doanh và thúc đẩy tinh thần khởi nghiệp.

- Các trường đại học cần có các chính sách khuyến khích, động viên tinh thần doanh nhân và khởi nghiệp của sinh viên, đặc biệt là sinh viên nữ thông qua việc tạo lập sân chơi phát triển ý tưởng kinh doanh và khởi nghiệp cho sinh viên.

Hai là, đẩy mạnh công tác tuyên truyền tinh thần doanh nhân và ý định khởi nghiệp cho sinh viên bằng các hình thức:

- Dành riêng một chuyên mục khởi nghiệp kinh doanh và tinh thần doanh nhân trên các phương tiện truyền thông đại chúng.

- Phát huy vai trò của các diễn đàn khởi nghiệp để lan tỏa tinh thần khởi nghiệp đến mọi công sở, trường học, mọi gia đình và cộng đồng xã hội.

- Phát hành các ấn phẩm về sinh viên khởi nghiệp; doanh nhân trẻ thành đạt; cẩm nang khởi nghiệp để truyền cảm hứng, định hướng và dẫn dắt tinh thần khởi nghiệp của sinh viên.

$\boldsymbol{B} \boldsymbol{a}$ là, hoàn thiện môi trường khởi nghiệp bằng cách:

- Chính phủ và TP.HCM cần tiếp tục kiến tạo môi trường khởi nghiệp cho sinh viên; khẩn trương bổ sung các ngành nghề mới, chưa có trong hệ thống ngành nghề được Chính phủ quy định, đồng thời xây dựng hệ sinh thái khởi nghiệp của từng ngành, từng lĩnh vực cụ thể và định hướng khởi nghiệp theo lĩnh vực đó.

- Phát triển và phát huy vai trò của các quỹ đầu tư khởi nghiệp và các vườn ươm khởi nghiệp, đồng thời có các chính sách khuyến khích và hỗ trợ sinh viên khởi nghiệp như cấp tín dụng lãi suất ưu đãi; chính sách miễn, giảm thuế thu nhập doanh nghiệp cho sinh viên khởi nghiệp trong những năm đầu; xây dựng các chương trình dự án kinh doanh cho sinh viên khởi nghiệp, v.v.

- Trung tâm Hỗ trợ thanh niên khởi nghiệp (BSSC) của Thành Đoàn TP.HCM cần phát huy hiệu quả của diễn đàn: "Không gian sinh viên khởi nghiệp" để hướng đến hình thành cộng đồng sinh viên khởi nghiệp cùng nhau hợp tác, chia sẻ, hỗ trợ và phát triển.

- Các trường đại học phải đóng vai trò quyết định trong việc tuyên truyền, giáo dục, trang bị kiến thức, kỹ năng và trải nghiệm để sinh viên sẵn sàng khởi nghiệp.

Bốn là, phát huy nỗ lực và bản lĩnh khởi nghiệp của sinh viên, các trường đại học kết hợp với cơ quan truyền thông tăng cường công tác giáo dục, tuyền truyền sinh viên nhận thức vai trò của tinh thần khởi nghiệp; những điều kiện để biến ý tưởng khởi nghiệp thành hiện thực, những rủi ro có thể phải đối mặt, để sinh viên có một thái độ đúng đắn đối với vấn đề khởi nghiệp, nuôi dưỡng niềm đam mê khởi nghiệp và xây dựng bản lĩnh tinh thần doanh nhân.

Năm là, đẩy mạnh và nâng cao hiệu quả hoạt động tư vấn khởi nghiệp. Trong đó, Trung tâm Hỗ trợ thanh niên khởi nghiệp (BSSC) của Thành Đoàn TP.HCM cần khẩn trương cho ra mắt Website cơ sở dữ liệu khởi nghiệp để sinh viên có điều kiện tiếp cận và tham vấn các mô hình khởi nghiệp; các yêu cầu, điều kiện khởi nghiệp, những rủi ro trong quá trình khởi nghiệp, từ đó nâng cao nhận thức kiểm soát hành vi khởi nghiệp của sinh viên. 


\section{Tài liệu tham khảo \\ Tiếng Việt}

Đỗ Thị Hoa Liên. (2016). Các yếu tố ảnh hưởng đến ý định khởi nghiệp kinh doanh của sinh viên Quản trị kinh doanh Trường đại học lao động xã hội (cơ sở TP.HCM). Tạp chí Khoa học Yersin, số 1(11/2016), tr. 44-52.

Phan Anh Tú và Giang Thị Cẩm Tiên. (2015). Nghiên cứu các yếu tố ảnh hưởng đến ý định khởi sự doanh nghiệp: Trường hợp sinh viên Khoa Kinh tế và Quản trị kinh doanh Trường Đại học Cần Thơ. Tạp chí Khoa hoc Trường ĐH Cần Tho; số 38 (2015), tr. 59- 66.

\section{Tiếng Anh}

Ambad, S. N. A and Ag Damit, D. H. D. (2016). Determinants of Entrepreneurial Intention Among Undergraduate Students in Malaysia. Procedia Economics and Finance, 37, 108 - 114.

Ajzen I., Fishbein, M. (1975). Belief, Attitude, Intention and Behavior: An Introduction to theory and research. Addition-Wesley, Reading, MA.

Ajzen, I. (1991). The Theory of Planned Behaviour. Organization Behavior and Human Decision Processes, No. 50, pp. 179-211.

Autio, E., Keeley, R.H., Klofsten, M., Parker, G.C. and Hay, M. (2001). Entrepreneurial Intent among Students in Scandinavia and in the USA. Enterprise and Innovation Management Studies 2 (2), 145-160.

Grimaldi, R. and Gradi, A. (2005). Business incubators and new venture creation; an assessmant of incubating models. Technovation, Vol. 25 No.2, pp. 111-121.

Karali, S. (2013). The Impact of entrepreneurship education programs on entrepreneurial intentions: An application of the theory of planned behavior. Erasmus University of Rotterdam - Master Thesis.

Khan, M. M., Ahmed, I., Nawaz, M. M., \& Ramzan, M. (2011). Impact of personality traits on entrepreneurial intentions of university students. Interdisciplinary Journal of Research in Business, 1(4), 51-57.

Lĩnán, F., Rodríguez - Cohard, F. J., Rueda-Cantuche, J. M. (2011). Factors affecting entrepreneurial intention levels: a role for education. International Entrepreneurship and Management Journal, Volume 7, Issue 2, pp195-218.

Luthje, C., \& Franke, N. (2003). The making of an entrepreneur: testing a model of entrepreneurial intent among engineering students at MIT'. $R \& D$ Management, 33, (2), 135-147.

Ooi, Y, K., Selvarajah, C., \& Meyer, D. (2011). Inclination towards entrepreneurship among university students: An empirical study of Malaysian university students. International Journal of Business and Social Social Science, 2 (4), 206-220.

Radas, S và Bozic, L. (2009). The antecedents of SME inovativeness in an emerging transition economy. Technovation, Vol. 29, pp. 438-450. 
Schaper, M. and Volery, T. (2004). Entrepreneurship and small business: A Pacific Rim perspective. Milton, Queensland, John Wiley and Sons Australia Ltd.

Shapero, A. and Sokol, L. (1982). The social dimensions of entrepreneurship. in Kent, C., Sexton, $D$. and Vesper, K. (Eds). The Encyclopedia of Entrepreneurship. Prentice-Hall, Englewood Cliffs, NJ, p. 72-90.

Tam, H. W. (2009). How and to What Extent Does Entrepreneurship Education Make Students More Entrepreneurial? A California Case of the Technology Management Program. Doctor of Philosophy Dissertation, University of California, Santa Barbara.

Venkatachalam, V. B. and Waqif, A. A. (2005). Outlook on integrating entrepreneurship in management education in India. Decision 32(2): 57-71.

Yordanova, D., \& Tarrazon, Maria-Antonia. (2010). Gender Differences in Entrepreneurial Intentions: Evidence From Bulgaria. Journal of Developmental Entrepreneurship, 15(3), $245-$ 261. 